\title{
«Tierra de nadie»: Estado y desarrollo humano en Madre de Dios*
}

\author{
Alejandra Fuentes**
}

* Basado en mi tesis de licenciatura (Facultad de Ciencias Sociales-Ciencia Política y Gobierno).

* Licenciada en Ciencia Política y Gobierno por la Pontificia Universidad Católica del Perú. Investigadora en The Network: Advocating Against Domestic Violence, en la ciudad de Chicago, EE.UU. Correo electrónico: alejandra.fuentes@pucp.pe

Recibido: 16/06/2019. Aceptado: 22/01/2020. 


\title{
«Tierra de nadie»: Estado y desarrollo humano en Madre de Dios
}

\section{RESUMEN}

Tradicionalmente, una de las preguntas más importantes en las ciencias sociales ha sido aquella que indaga por las diferencias en los niveles de desarrollo (entre regiones, países y unidades subnacionales). Sin embargo, solo en las últimas décadas se ha empezado entender el desarrollo desde una perspectiva centrada en las personas y, particularmente, en su bienestar y libertad para vivir la vida que elijan vivir. En esta línea, el presente artículo indaga por qué una región como Madre de Dios — caracterizada por su complejidad geográfica, economías ilegales, altos índices de trata de persona y otras problemáticas - ha logrado mantener en el tiempo niveles relativamente exitosos en el índice de desarrollo

humano. Desde una perspectiva subnacional, se plantea que en dicha región coexisten tres niveles diferenciados de desarrollo humano en tres zonas distintas del territorio que son eclipsados por la medición regional. Estos tres niveles diferenciados, a su vez, son el resultado de distintos patrones de relaciones entre Estado y sociedad basados en dos condiciones: (1) el tipo de actores no estatales y (2) la naturaleza de la interacción.

Palabras clave: Amazonía, desarrollo, Estado, territorio, actores.

\section{«No one's territory»: State and human development in Madre de Dios}

\begin{abstract}
Traditionally, one of the most important questions in social sciences has been one that asks about differences in levels of development (between regions, countries and sub-national units). However, it is only in the last decades that development has begun to be understood from a perspective centered on people and, particularly, on their well-being and freedom to live the life they choose to live. This article argues why a region like Madre de Dios-characterized by its geographical complexity, illegal economies, high rates of human trafficking and other problems- has managed to maintain relatively successful levels in the Development Index over time. Human. From a sub-national perspective, it is proposed that in this region three different levels of human development coexist in three different areas of the territory that are overshadowed by regional measurement. These three differentiated levels, in turn, are the result of different patterns of relations between State and society based on two conditions: (1) the type of non-state actors and (2) the nature of the interaction.
\end{abstract}

Keywords: Amazon, development, state, territory, actors. 


\section{INTRODUCCIÓN}

La construcción del Estado peruano ha tenido, posiblemente, uno de los recorridos más ásperos en la historia del Perú. Mientras que la consolidación de los Estados-nación en Europa tenía que ver con el éxito bélico, los retos enfrentados por el Estado peruano, en su intento por ocupar su propio territorio significaron un esfuerzo de mayor envergadura. Los principales enemigos: una geografía rebelde y grupos nativos enraizados (Morel, 2014).

Así pues, la Amazonía significó un desafío mayor que el resto del territorio peruano. Si bien es abundante en recursos naturales, tales como los minerales, la madera y la biodiversidad, se caracteriza también por el desarrollo de economías ilegales, encontrarse particularmente lejana del centro político, mantener altos índices de trata de personas, entre otras problemáticas. La expansión del imperio de la ley, por lo tanto, fue restrictiva y circunstancial.

Madre de Dios no fue una excepción a la regla y la conquista del Estado por su propio territorio significó un reto y aunque los límites fronterizos ya están delimitados, la presencia del Estado en la región sigue siendo escasa en Madre de Dios (Fuentes, 2018). No obstante, en la reciente historia (1998-2012), Madre de Dios ha mostrado niveles muy altos de desarrollo humano en comparación con el promedio nacional y ha ocupado uno de los primeros puestos según la medida más conocida del fenómeno: el índice de desarrollo humano (IDH) del Programa de las Naciones Unidas para el Desarrollo (PNUD). Más aún, se ha ubicado en un nivel superior a los demás departamentos amazónicos (Ucayali, Amazonas, Loreto y San Martín) de manera sostenida (PNUD, 2012), a pesar de compartir con ellos una situación económica, social y política bastante similar (Barrantes y Glave, 2014; Cotler, 2009).

El presente artículo propone que una mirada exhaustiva al buen desempeño en materia de desarrollo humano de esta región revela que dicho desempeńo responde solo a una zona geográfica específica dentro de dicha región, mientras que las otras partes del territorio experimentan una realidad notablemente distinta en cuanto a la calidad de vida (Mujica, 2014; Vallejo, 2014). De manera más precisa, se propone que en la región coexisten tres niveles distintos de desarrollo humano: una zona urbana, como centro económico y político de la región, la cual goza de un nivel de desarrollo alto y desigual; una zona rural y desatendida, con un nivel medio de desarrollo humano pero uniforme; y, por último, la zona de La Pampa, donde la posibilidad de acceder a un nivel de vida digno es muy baja, aunque desigual. Aunque estas variaciones no responden a la división político-territorial formal del país, constituyen patrones contrastantes de desarrollo humano que vale la pena destacar.

Teniendo como objetivo responder cuáles son los factores que explican el establecimiento y coexistencia de estas tres zonas con diferentes niveles de desarrollo humano en la misma región amazónica, se propone lo siguiente: las distintas configuraciones 
de relaciones entre el Estado y la sociedad civil organizada tienen como producto distintos niveles de desarrollo humano, los cuales se reflejan en las zonas de dicha región. Para ello, será necesario analizar un patrón regional de desarrollo humano que cambia a nivel intrarregional e identificar aquellos factores que explican la existencia de diferentes niveles de desarrollo dentro del territorio de Madre de Dios. A su vez, esta investigación busca explicar cómo la existencia de este patrón de desarrollo y los factores que lo hacen posible están a la base del posicionamiento de Madre de Dios a nivel nacional y, en particular, entre los departamentos amazónicos en términos de los resultados obtenidos en las mediciones más recientes del IDH, del cual se harán las respectivas precisiones (definición y conceptualización) en la sección uno.

Como se dijo previamente, el desarrollo de esta investigación depende de dos acciones concretas. Primero, la evidencia de la diferenciación de desarrollo humano de las zonas y segundo, la explicación de este fenómeno a partir de las relaciones Estado-sociedad. Entre los hallazgos, el estudio reveló que existen tres zonas con distintos niveles de desarrollo humano. La primera zona, con el nivel más alto, es la zona urbana, ubicada en la capital Puerto Maldonado. Los indicadores referentes a esta zona señalan que las personas pueden vivir de manera digna y alcanzar su libertad. La zona de minería ilegal (La Pampa) y la zona rural no experimentan lo que la zona urbana, dado que los resultados son bajos e inferiores al resto del país, incluso dentro de la región. En el primer caso, encontramos altos ingresos económicos, dado que los ingresos se justifican por los ingresos de la minería ilegal y a su vez, resultados con respecto a salud y educación muy bajos, lo que coloca a esta zona en el peor puesto de desarrollo humano entre las tres. En cuanto al segundo caso, si bien los indicadores no son tan favorables, se comprueba que las posibilidades de vida no son tan limitadas, por lo que esta zona responde a un nivel medio, entre las zonas previamente descritas.

En cuanto a la explicación de este fenómeno territorial de desarrollo humano, el trabajo argumenta que la diferenciación en los niveles de desarrollo humano en Madre de Dios se explica a través de la interacción entre Estado y sociedad, la cual varía en cada una de las zonas territoriales dentro de la región. En la zona urbana, el Estado no encuentra problemas para asegurar un adecuado desarrollo humano, brindando servicios de salud y educación a un sector económicamente integrado al resto del país; en la zona rural, la desatención se sustenta en la falta de interés del Estado, al ser esta una zona de baja y compleja densidad poblacional y geografía accidentada; y finalmente, en La Pampa, el Estado encuentra actores desafiantes en el territorio, que dificultan su intervención para asegurar el desarrollo humano. El presente artículo demuestra que, dependiendo de la relación entre el Estado y la sociedad en cada una de las zonas, se gestan distintos niveles de desarrollo humano en la misma región.

Dada la complejidad del argumento, se consideró necesario realizar un estudio cualitativo centrado en el departamento de Madre de Dios, con la finalidad 
de conocer a profundidad la situación de las principales zonas de este territorio regional. Asimismo, si bien esta investigación califica como un estudio subnacional, se estudiaron unidades subnacionales alternativas, alejándose de las divisiones administrativas (regional, provincial, etc), para centrarse «zonas», delimitadas de acuerdo al nivel de desarrollo humano (Giraudy, Moncada y Snyder, 2019). La evidencia empírica que brinda sustento al argumento se basa no solo en un trabajo de gabinete (cifras oficiales, recursos bibliográficos, artículos periodísticos y otras fuentes secundarias), sino también en un trabajo de campo realizado en la ciudad de Puerto Maldonado y alrededores, entre febrero y octubre del año 2017 durante dos semanas cada visita. Este consistió en veinte entrevistas semiestructuradas a altos funcionarios y a profundidad a funcionarios de rango medio (pertenecientes al gobierno regional y gobierno central en la región) y actores de la sociedad civil organizada con incidencia en los sectores de salud y educación. De esta manera, se obtuvo una perspectiva, debidamente triangulada, de cada uno de los actores pertenecientes al Estado y sociedad civil. En el caso de los altos funcionarios, se obtuvo una perspectiva institucional sobre cómo se busca proveer de desarrollo humano (servicios de salud y educación desde el Estado). Para caracterizar de manera más acertada cómo funciona la provisión de los servicios, se entrevistó a funcionarios de rango medio. Se utilizó la misma lógica para las entrevistas de los actores pertenecientes a la sociedad civil, para conocer su perspectiva, debidamente triangulada posteriormente.

El documento está organizado de la siguiente manera: primero, se abordarán algunas aproximaciones teóricas que permitan comprender el argumento planteado; segundo, se realizará una caracterización de la región, la cual permitirá evidenciar la existencia de las tres zonas de desarrollo humano; tercero, se buscará explicar cómo las relaciones Estado-sociedad inciden en el nivel de desarrollo humano. Por último, se esbozan unas conclusiones, alcances y limitaciones de la investigación.

\section{AProximaCiONES TEÓRICAS: RELACIONES ESTADO Y SOCIEDAD}

La presente sección ofrece una breve revisión de distintos estudios que se relacionan o se han centrado en las relaciones Estado-sociedad, pues las distintas configuraciones de estas relaciones logran explicar el nivel de desarrollo humano en cada una de las tres zonas de la región. La revisión sugiere que una de las configuraciones más influyentes en el nivel de desarrollo humano es la presencia de actores no estatales en dos variantes: la coalición, figura en la que las instituciones del Estado y la sociedad civil organizada actúan en coordinación para proveer servicios sociales a la población. El conflicto, por su parte, propone la idea de un enfrentamiento entre el Estado y «desafiantes territoriales» quienes se oponen a la intervención del primero en el territorio (Sabatier y Jenkins-Smith, 1993; Migdal, 1994; Eaton, 2012; Slater 
y Kim, 2015). Estas configuraciones condicionan al Estado en cuanto a su intervención, generando preferir no intervenir o tratar de intervenir sin éxito.

En primer lugar, es necesario definir el concepto de desarrollo humano. Siendo este bastante amplio, esta investigación tomará como definición la propuesta de PNUD del año 2004, la cual abarca las clásicas dimensiones de ingresos económicos, salud y educación como claves puedan alcanzar sus aspiraciones de una vida digna y, además, tiene un enfoque territorial, en donde el Estado tiene un rol activo para el logro de estas aspiraciones: «El desarrollo humano se trata sobre todo de ampliar las opciones de la gente, es decir, permitir que las personas elijan el tipo de vida que quieren llevar, pero también de brindarle tanto las herramientas como las oportunidades para que puedan tomar tal decisión» (PNUD, 2005, p. 36) y se partirá de la definición básica de PNUD para establecer las dimensiones: «[1] [...] una vida larga y saludable, medida por la expectativa al nacer; [2] el conocimiento, medido por la tasa de alfabetización de adultos (con una ponderación de dos tercios) y la tasa bruta combinada de matrícula en escuelas primarias, secundarias y terciarias (con una ponderación de un tercio); y [3] un nivel de vida digno, medido por el PIB per cápita en términos de paridad del poder adquisitivo (PPA) en dólares estadounidenses» (Mancero, 2005, p. 6).

El IDH se construye a partir de cada dimensión y tiene un puntaje del 0 al 1 , siendo 0 el mínimo y 1, el máximo. Si bien este índice es utilizado a nivel mundial, dada la precisión que tiene, resulta limitado para esta investigación, dado que esta es un estudio de caso y lo que se busca es profundizar y no generalizar (Gerring, 2008). Por lo tanto, se proponen dos criterios con respecto a la variable dependiente: se utilizan las mismas tres dimensiones señaladas, pero se agregan nuevos indicadores que se suman a los anteriores, los que permiten que una caracterización de las zonas observadas, para que esta sea más acertada. Un nivel de desarrollo es considerado como «alto» cuando los indicadores referentes a la zona arrojan resultados altos (superiores al promedio nacional o en comparación con otras zonas) en todas las dimensiones. Si la zona presenta resultados favorables solo en los indicadores de una o ninguna dimensión, se considerará una región con un desarrollo humano «bajo». Asimismo, se reconoce que los niveles describen patrones regionales, por lo tanto, puede existir desigualdad en el nivel de vida de los habitantes de una misma zona. Para ello se plantea ańadir la homogeneidad como parte de la caracterización. Así, se puede clasificar una zona con un nivel de desarrollo humano «alto» y homogéneo, en donde los habitantes, a excepción de algunos, acceden a un nivel de vida que les permite la posibilidad de desarrollarse en un ambiente favorable para ampliar sus posibilidades.

Sobre las relaciones Estado-sociedad, será necesario tener en cuenta la complejidad de estas, pues el primero está compuesto también por ciudadanos y 
ciudadanas, con sus propias necesidades familiares (como funcionarios/as). La segunda, refiere a un sector de la sociedad organizado en colectivos u organizaciones, diferenciado de la población en general (Dargent y Urteaga, 2016; Migdal, 1997; Vergara, 2015). Sobre las relaciones Estado-sociedad, existen autores que han enfatizado que la coalición entre actores de ambos bandos suele estar vinculada a prácticas particularistas (Altman, 2012), este estudio demuestra que en algunos casos estas relaciones pueden lograr mejoras en el bienestar de la población. Asimismo, los actores no estatales no necesariamente actúan al margen del Estado o como sustitutos, sino que algunos actúan como complementos y puedan ser parte de una dinámica de cooperación (Hale, 2006; Geddes, 1994). Por el momento, esta simplificación resulta teóricamente útil.

Una de las características de la definición de desarrollo humano adoptada (PNUD, 2016) para este trabajo es la importancia que le da al rol del Estado. Si bien el desarrollo humano se enfoca en las personas, su bienestar y libertad, esta definición debate sobre quién se asegura del desarrollo de las personas y dónde debe darse. Precisamente allí talla el Estado como el que asegura el bienestar en dicho espacio (PNUD, 2009). Este rol se materializa a través de este como proveedor de servicios sociales, tales como la salud y la educación (contempladas como dimensiones dentro de la definición) para promover oportunidades igualitarias (Pribble, 2011).

En un escenario ideal, el Estado sería capaz de proveer de servicios, de manera homogénea, a quienes pertenecen su territorio y así asegurar el bienestar de sus ciudadanos y ciudadanas. Sin embargo, como se postuló previamente, los niveles de desarrollo humano varían a lo largo de la región, lo que indica una provisión de dichos servicios de manera heterogénea. ¿A qué se debe esto? Más allá de la capacidad estatal, las relaciones entre Estado y sociedad juegan un rol fundamental, dado que, al variar a lo largo de la región, condicionan la intervención del Estado (O’Donnell, 1993; Herbst, 2000; Eaton, 2012; Amengual, 2014).

Para lograr complejizar el diálogo, es necesario tener en cuenta algunas definiciones. Mientras, Soifer (2008) y Soifer y vom Hau (2008) proponen que la capacidad estatal abarca el alcance territorial, la autonomía frente a actores no estatales y la profesionalización de la burocracia (Giraudy, 2012, p. 601), el caso de Madre de Dios permite cuestionar estos preceptos. Mann (2008), plantea un escenario en donde distintos actores (entre ellos, el Estado y la sociedad) interactúan y a través de un concepto denominado poder infraestructural se ejerce poder en el territorio, a través de las funciones que el Estado realiza. Así, un Estado capaz es aquel que, por ejemplo, mantiene seguros a sus ciudadanos y ciudadanas, dentro de un marco de leyes que delimite su comportamiento y los provea de servicios que satisfagan sus necesidades básicas. En un caso contrario, el Estado también podría no proveer de servicios en ciertas partes de su territorio. 
Como respuesta a esto, O'Donnell (1993) postula que la presencia del Estado es heterogénea, lo que puede visualizarse en el cumplimiento de la ley en distintas partes de un país. ¿Esto sería incapacidad del Estado? La propuesta de Herbst (2000) permite explicar esta variación a través de su trabajo acerca de la construcción del Estado en África y justifica las propuestas de Soifer y vom Hau (2008). De acuerdo con el autor, los líderes enfrentan costos cuando buscan expandir su autoridad y en esta expansión existen algunas zonas que representan más esfuerzo que otras. En la misma línea, Boone (2012) plantea que esto tiene que ver con que el Estado evaluará la proyección de su poder de acuerdo con costos y beneficios. Es decir, si alguna zona es de sencilla accesibilidad, tanto geográficamente, como socialmente, además de brindarle beneficios, el Estado intervendrá. En el caso contrario, no o tomará más tiempo hacerlo.

Claro está, esta intervención se relaciona directamente con la provisión de servicios sociales del Estado, estando estos atados a su intervención. Por ejemplo, en el caso que una comunidad indígena organizada tenga problemas para relacionarse con el gobierno y busquen la defensa de su territorio por sobre cualquier negociación (Ziegler-Otero, 2009; Selverston, 1994, p. 13; Chartock, 2013), la intervención del Estado se vería desacelerada, por evitar conflictos con quienes se encuentran en dicho territorio. Se puede decir entonces que la relación entre Estado y sociedad puede ser, tanto porosa y conflictiva, como armoniosa y colaborativa.

En la primera variante, los actores no estatales entienden al Estado y sus instituciones como enemigo e incluso puede ser también viceversa. De esta manera, los actores desafían las decisiones que el Estado toma en relación con el territorio (Eaton, 2012), oponiéndose férreamente a ellas e incluso no permitiendo que este tome decisión alguna (Scott, 1990; Holland, 2016). Así, encuentran formas de resistencia diaria, a través de la oposición del Estado (Scott, 1990). Para intereses de este estudio, se hará uso de la definición de este autor sobre los desafiantes territoriales: «son grupos sociales o actores no estatales que se desarrollan en espacios no controlados por el Estado, lo que suele indicar la ausencia de eficiencia de la policía o de la presencia militar» (Eaton, 2012, p. 648).

¿Cuál es el efecto de esta resistencia? Como se mencionó previamente, el Estado evalúa sus posibilidades y dependiendo del beneficio, decide no intervenir. Siguiendo esta lógica, Slater y Kim señalan que el Estado, buscando minimizar sus esfuerzos, opta por no intervenir, más allá si tiene la capacidad de hacerlo (Holland, 2016).

En el caso de la segunda variante, la relación entre Estado y sociedad puede ser también de cooperación: la sociedad civil organizada y el Estado coordinan y mientras este recibe las demandas y logra canalizarlas en efectivas políticas públicas implementadas en el territorio este responde con su aprobación e incluso con su apoyo (Eaton, 2012; Migdal, 1994). A este tipo de relación se le llama coalición, 
la cual está conformada por actores pertenecientes a organizaciones de la sociedad civil e incluso del Estado, con una agenda alineada con creencias básicas y acciones que guían a un mismo objetivo (Sabatier y Jenkins-Smith, 1993; Augusto, 2016). En este caso, el efecto resulta favorable, pues el costo que enfrenta el Estado es bajo y en caso los beneficios sean altos, el interés será mayor aún.

En resumen, más allá de la capacidad estatal, el Estado condiciona su intervención de acuerdo a la relación establecida con la sociedad civil. Mientras que la relación puede ser de cooperación y de coordinación, también puede ser conflictiva, lo que genera incentivos nulos. ¿Cómo afectan dichas relaciones el desarrollo humano de manera diferenciada en las zonas de Madre de Dios? Como se dijo anteriormente, la provisión de servicios sociales (salud y educación) está incluida dentro de la intervención estatal y siendo esta distinta, de acuerdo con la relación Estadosociedad en cada zona, por lo que el impacto en la población también será distinto.

\section{3. ¿DEsarrollo humano en Madre de Dios? Caracterizando una región DISPAR}

Esta sección tiene como objetivo evidenciar la existencia de tres zonas con distintos niveles de desarrollo humano en la región de Madre de Dios: la zona urbana, la zona rural y la zona de La Pampa. Cabe recalcar que el presente estudio abarca la región en el período 2007-2017.

\subsection{Características generales del desarrollo humano de la región y delimitación espacio tiempo}

El desarrollo humano, como se ha visto previamente, es un concepto complejo por lo que se idearon distintas formas de medirlo. La manera más tradicional de medición es el índice de desarrollo humano, el que, independientemente del contexto, debería ilustrar cómo es el desarrollo humano. Si bien existen muchos cuestionamientos sobre la capacidad de lograr su objetivo de generalizar y lograr brindar una medida (Albina, 2011; Lindberg, Coppedge, Gerring y Teorell 2014), se aplica a Perú y es un referente de desarrollo humano. Sus resultados se exponen en el índice de desarrollo humano.

El informe del año 2000, detalló que Perú se hallaba en el puesto 75 de 188 países, con un IDH de 0,677 (siendo el máximo el 1 y el mínimo el 0). Diez años después, en el año 2010, si bien el IDH aumentó a 0,721, Perú se posicionó en el puesto 90, dado que el incremento también afectó a otros países. Para el año 2015, subió al puesto 87 y el puntaje aumentó a 0,74. Asimismo, a nivel regional, los departamentos de Lima, Arequipa y Moquegua lideraron el puntaje desde el primer informe realizado a nivel subnacional. 


\section{Gráfico 1. IDH comparado (Madre de Dios)}

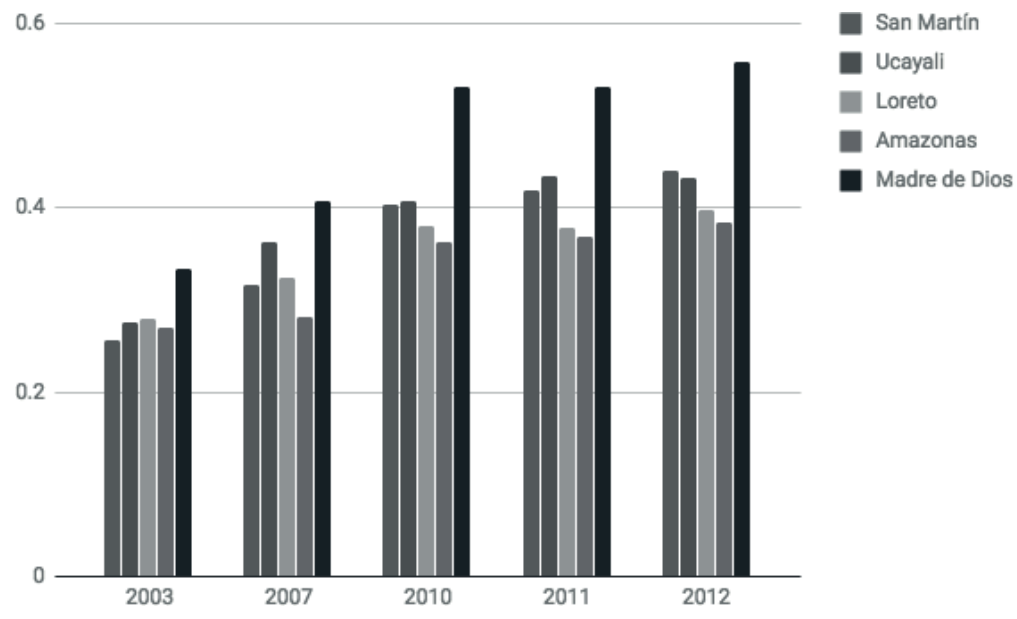

Fuente: PNUD. Elaboración propia.

En comparación con sus regiones pares amazónicas (Amazonas, San Martín, Loreto y Ucayali), estas comparten una trayectoria tanto económica como social y demográfica, lo que permite que se las haya podido estudiar agrupadas. Sin embargo, de acuerdo con el Gráfico 1, solo la región Madre de Dios posee resultados distintos, al tener un puntaje superior al de sus pares. De hecho, de acuerdo con el Mapa 1, Madre de Dios se asemeja a las regiones de la costa. Como se observa, a nivel provincial (en el mapa de todo el país), las provincias de Madre de Dios presentan niveles similares a las provincias de la costa, de igual manera que en el caso del otro mapa, a nivel distrital.

De esta manera, se puede asegurar que el nivel de desarrollo humano (IDH) de Madre de Dios se asemeja al de regiones más desarrolladas que el resto. En perspectiva temporal comparada, el promedio nacional se se sostiene desde el ańo 2002 hasta 2007, en donde se dispara, como se puede ver en el Gráfico 2. Al contrastar los datos oficiales con los estudios empíricos cualitativos, detalla que el desarrollo humano en la región es bastante complejo.

La situación de Madre de Dios, hasta el año 2017, año en que se culminó esta investigación, está caracterizada por una serie de aspectos demográficos, económicos y sociales que permiten comprender el desarrollo humano en la región ${ }^{1}$. Para ese año, la población censada fue de 141070 habitantes, lo que representa el 0,5\% de la población nacional (Censo 2017), siendo la tasa de crecimiento anual de la población ha disminuido en los últimos diez años. Una característica particular de Madre de Dios es que alberga casi el 3\% de la población indígena del país, entre ella, comunidades no contactadas, que sumarían 1500 personas, lo que confirma la presencia de diversidad étnica en la región.

1 Datos tomados del Instituto Nacional de Estadística e Informática. 


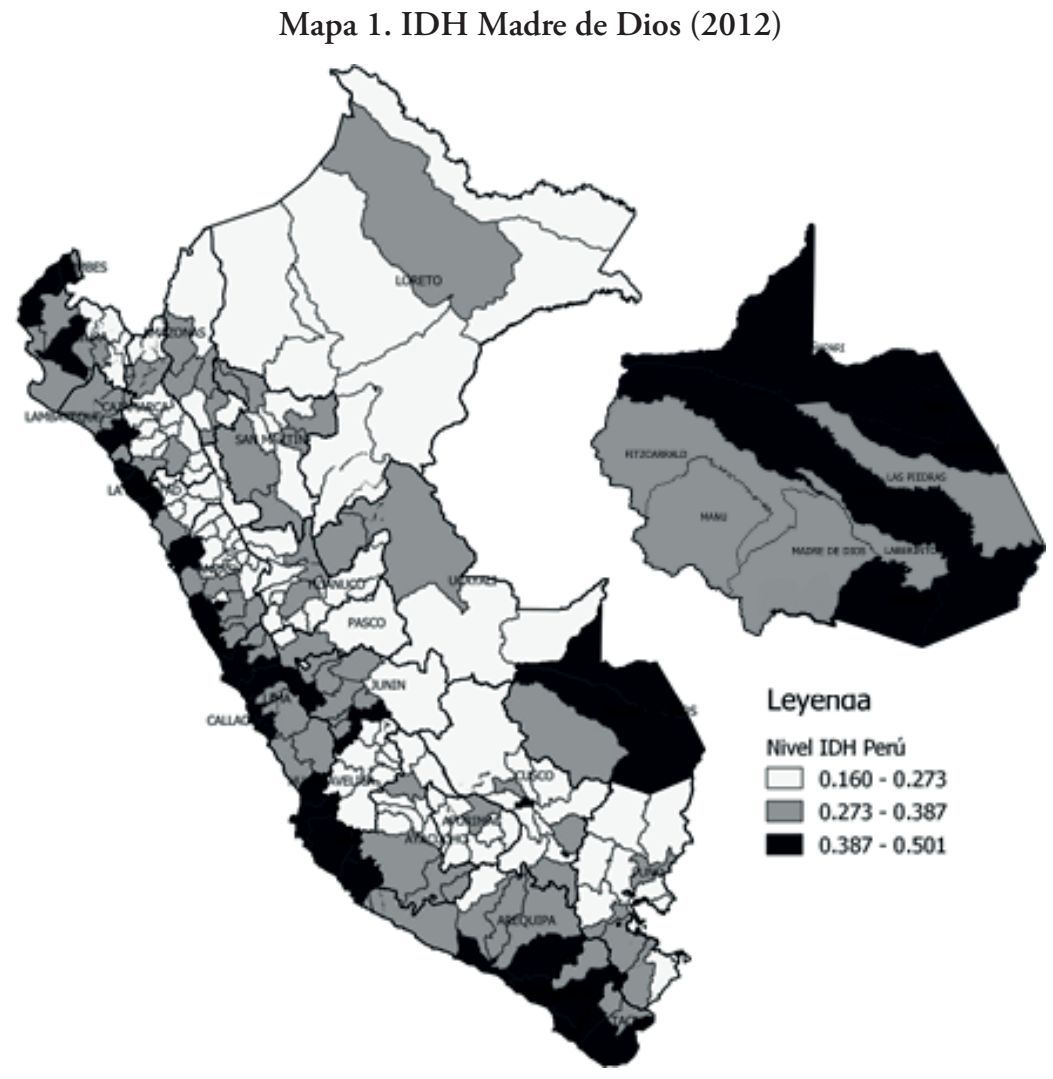

Fuente: PNUD, 2012. Elaboración propia.

Gráfico 2. IDH comparado (Madre de Dios)

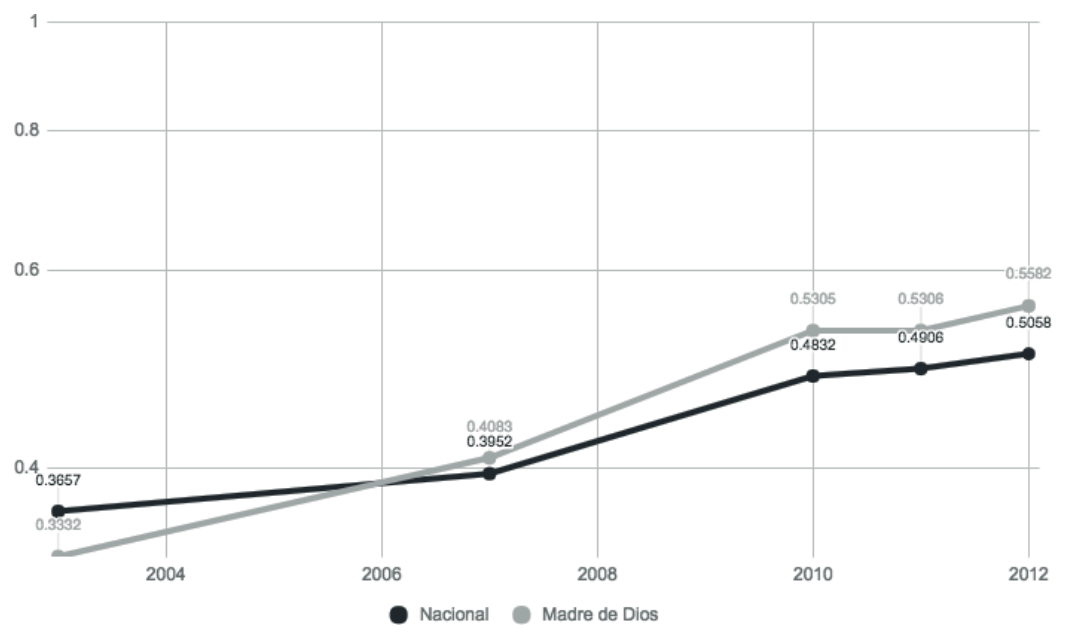

Fuente: PNUD. Elaboración propia. 
En el aspecto económico, Madre de Dios destaca por ser una región muy rica en recursos. Para el año 2016, esta obtuvo un producto bruto interno (PBI) regional de 2726380 miles de soles, de los cuales 1292704 se deben al sector minero, el mismo que aporta económicamente 6887 miles de soles a la economía nacional (Ministerio de Economía y Finanzas, 2017). No obstante, el canon minero fue bastante bajo frente al resto de regiones mineras del país, dado que gran parte de esta actividad es de corte ilegal (6,89 millones de soles).

En el plano político, Madre de Dios siempre fue una región que optó por líderes outsiders (no políticos tradicionales, sino empresarios o mineros), los cuales mantenían relaciones conflictivas con el gobierno ejecutivo ${ }^{2}$ (principalmente con respecto a la actividad minera). El gobernador de la gestión anterior, Luis Otsuka, se caracterizó por mantener una relación hostil con el Poder Ejecutivo y defender la actividad minera ilegal, perfil similar al de su asesor (2010-2014), quien está siendo procesado por corrupción. Parte del escenario político también está caracterizado por la participación de, por un lado, las comunidades nativas, congregadas en la Federación Nativa del Río Madre de Dios y Afluentes (Fenamad), como principal organización indígena, en oposición a la actividad minera a escala masiva, dado que esta afecta directamente el medio ambiente (la reserva Tambopata) y su propia actividad (ellos lo hacen de manera artesanal) (Orihuela, 2014). Por otro lado, destaca también la Federación Minera de Madre de Dios (Fedemin) y su defensa de la actividad minera en la región, al margen de su carácter informal e ilegal.

En el plano social, los indicadores de educación, salud y pobreza permiten complementar el panorama sobre el desarrollo humano en Madre de Dios. En primer lugar, la pobreza es inferior al resto de regiones, habiendo llegado, para 2015, a $7,1 \%$ (pobreza monetaria). Esto contrasta con las regiones amazónicas Amazonas, Loreto, San Martín y Ucayali, que inciden en $42,3 \%$, 35\%, 27,6\% y 11,4\%, respectivamente. La salud, por su parte, goza de niveles similares al resto de regiones en la mayoría del período estudiado, pero ya en el último año superó la tasa de mortalidad neonatal a nivel nacional en cinco puntos porcentuales con respecto al año pasado, lo que indica un avance a nivel regional sobre el promedio de todas las regiones. Por último, la educación posee niveles superiores al resto de regiones, dato que se sustenta en la baja tasa de analfabetismo de la población de quince ańos a más para el año 2015 (3,8\%). En suma, Madre de Dios se posiciona como una región en vías de superar a las regiones costeras, puesto que su desempeño ha ido mejorando a lo largo de los ańos y estos indicadores denotan un claro desarrollo socioeconómico, lo que sustenta también el índice de desarrollo humano.

2 Véasehttps://ojo-publico.com/Luis-Otsuka-El-gobernador-minero-que-quiere-poner-en-jaque-a-PPK 


\subsection{Zonas de desarrollo humano}

Con respecto al bienestar de las personas, como se dijo previamente, esta subsección lo que busca es demostrar que existen estas tres zonas de desarrollo humano, a partir de las dimensiones establecidas por el índice de desarrollo humano, además de la información previamente presentada e información más precisa y adecuada para dichas dimensiones.

Como se dijo previamente, esta categorización está basada en las dimensiones propuestas por el IDH: una vida larga y saludable, referida a indicadores de salud; conocimiento, que refiere a indicadores de educación; y un nivel de vida digno, que refiere al capital de las familias. Cada dimensión cuenta con un número de indicadores específicos propuestos por PNUD.

La suma entre los indicadores de cada zona y los estudios empíricos brindarán una caracterización para establecer distintos niveles de desarrollo humano. Para ello, como se mencionó previamente, hay que tomar en cuenta lo siguiente: primero, se ańadieron otros indicadores, algunos jamás contemplados por PNUD, pues son muy particulares de la región y necesarios, si se busca especificidad en vez de generalizar (Sartori, 1994). Segundo, se tomó en cuenta la homogeneidad dentro de la región, lo que deja explicar zonas en donde hay un alto desarrollo humano pero algunas zonas en donde no.

En Madre de Dios, entonces, se reconocen tres niveles de desarrollo humano diferenciados, en cada una de las zonas: la zona urbana, con un nivel alto y desigual; la zona rural, con un nivel medio y uniforme; y la zona de La Pampa, con un nivel bajo y desigual. Las fuentes utilizadas incluyen indicadores, estudios de caso y trabajo de campo. Para cualquier referencia sobre la presente sección, ver el Anexo 1: metodología del desarrollo humano en Madre de Dios.

A continuación, se presentan las tres zonas incluidas en este estudio:

La zona urbana comprende a Puerto Maldonado, una ciudad que alberga 78378 habitantes (INEI, 2016), lo que sería el 57\% de la población madrediosina y que además es considerada la única ciudad de la región (a excepción de Ińapari e Iberia, pero entre ellas no suman más de once mil habitantes). Su extensión y ubicación (es atravesada por la carretera Interoceánica), lo que la convierten en el centro económico y político de la región. Asimismo, la actividad principal en esta zona es la del comercio.

Hablamos de una zona con un desarrollo humano alto y parejo en la zona urbana debido a que la zona arroja resultados altos en los indicadores de las tres dimensiones propuestas. En primer lugar, en el caso de la dimensión de «una vida larga y saludable», según los datos recogidos por PNUD, para el año 2012, la esperanza de vida al nacer era de 80,23 ańos, dato que supera al promedio nacional en seis años. Al contrastar con otros indicadores, se comprueba que en la zona, efectivamente, 
el ámbito de salud contribuye al desarrollo humano de las personas. Esta mejora se debe al crecimiento de la oferta médica en la región, pues ha habido un incremento del personal profesional en los últimos años ${ }^{3}$. Por otra parte, en 2011 se registró que un $80 \%$ de la población accede a fuentes mejoradas de agua, lo que contrasta con otras zonas, afectadas por la contaminación de mercurio.

En segundo lugar, para la dimensión de conocimiento, se utilizaron datos proporcionados por el IDH-PNUD e indicadores complementarios. En el caso de los primeros, la población con educación secundaria completa en la zona es de $72,21 \%$ y los años de educación de la población de 25 años a más es de 10,71 años, lo cual en ambos casos resulta bastante positivo, dado que superan el promedio nacional. En la línea de estos resultados, los indicadores complementarios mostraron lo siguiente: la población de 14 años a más sin nivel educativo es de $10 \%$, cifra por debajo del promedio nacional. No obstante, en las pruebas de la Evaluación Censal (ECE) de matemática, dirigida a alumnos de segundo de secundaria, solo el 7,90\% de la población urbana obtuvo un nivel satisfactorio. De la misma manera, la matrícula en el sistema educativo para la zona urbana fue de 37\%, un dato alarmante sobre el acceso a la educación en la región.

Aún así, la zona urbana destaca a nivel territorial, regional y nacional. Encontramos un colegio emblemático en Puerto Maldonado y demás colegios con una infraestructura sólida. No obstante, al no haber aumentado el sueldo, los profesores no tienen incentivos para mejorar la calidad de enseñanza ${ }^{4}$, lo que explica los resultados de la ECE. De esta manera, el nivel de desarrollo humano de la zona urbana se clasifica como alto, pero desigual/heterogéneo, lo que explica la disparidad en algunos indicadores. Esto quiere decir que existen barrios en en donde los indicadores pueden variar.

En tercer lugar, la dimensión de nivel de vida digno es netamente económica y uno de los indicadores por excelencia es el de ingreso familiar per cápita. El ingreso para esta zona para el año 2012, proporcionado por PNUD, fue de 1053, cifra que excede mínimamente al promedio de la región y en gran medida al promedio nacional, que bordeaba los 700 soles. Ahora, esta percepción de sueldos es exclusiva de los sectores minero, manufactura y comercio 5 . Esto quiere decir que las personas que no se dedican a esta actividad económica perciben sueldos que distan del monto indicado como promedio de la zona, lo que también indica desigualdad en esta dimensión.

\footnotetext{
3 Entrevista a Roy Santos, director Red Salud. Puerto Maldonado, 23 de febrero de 2017.

4 Entrevista a César Guadalupe, director de la Asociación Huarayo. Puerto Maldonado, 27 de febrero de 2017; entrevista a Vanessa Soto, coordinadora del proyecto de educación ambiental de la Asociación para la Conservación de la Cuenca Amazónica. Puerto Maldonado, 25 de febrero de 2017. 5 Entrevista a Flor Reátegui, directora de Trabajo y Promoción del Empleo. Puerto Maldonado, 22 de febrero.
} 
En resumen, nos encontramos con una zona que presenta un nivel alto pero desigual de desarrollo humano. Sobre los indicadores seleccionados, se puede decir que esta zona cumple con las expectativas de los indicadores a nivel regional, pues estos posicionan a Madre de Dios como una región con un desarrollo alto.

La zona rural tiene nivel de desarrollo humano medio y parejo/homogéneo. A diferencia de la urbana, no está concentrada en una ciudad, sino está compuesta por distintos distritos, en su mayoría alejados de la carretera Interoceánica ${ }^{6}$. La esperanza de vida al nacer, considerada por PNUD para su propio análisis, es de 75,31 años, cifra que sigue por encima del promedio de la región de Madre de Dios y del nacional, lo cual indica cierto nivel de bienestar. Sin embargo, el acceso a fuentes mejoradas de agua es únicamente exclusivo para el 32\% de la población. Asimismo, se registró en la zona rural contaminación por mercurio, la cual afectaba principalmente a las poblaciones indígenas de la región, debido a su consumo de pescado de río. El año pasado, la región estuvo en emergencia de salud debido a este problema (Gestión, 2016), que tiene origen en la minería artesanal.

Por su parte, los indicadores de la dimensión de educación no contrastaron tanto entre sí. Según los indicadores que utiliza PNUD, para el año 2012, la población con educación secundaria completa es de 43,06\% y los años de educación tienen un promedio de 8,3 años, cifras que están por debajo del promedio de la región, pero no muy alejadas del nacional. No obstante, observar otros indicadores educativos resulta desalentador: en cuanto a las pruebas ECE, aplicada a alumnos de segundo de secundaria, en 2016, el porcentaje de alumnos que alcanzó el nivel suficiente para la prueba de matemática fue el 2,9\% a nivel rural, cifra que solo difiere en cinco puntos porcentuales del porcentaje alcanzado por alumnos a nivel urbano. Esto se relaciona con el limitado acceso a la región, lo que condiciona el aprendizaje de los/as estudiantes ${ }^{7}$. Así, queda claro que el nivel educativo es inferior al urbano.

En cuanto a la dimensión económica, el indicador de ingreso familiar per cápita es de 814,63, ingreso que está por encima del promedio nacional por casi 200 nuevos soles (PNUD, 2013). Aun así no es suficiente para afirmar que esta zona es de un nivel de desarrollo humano alto, pero en contraste con la zona La Pampa, tampoco es baja, por lo que se le asigna a la zona rural un nivel medio, además porque muchos resultados están cercanos a los promedios nacionales.

La Pampa está clasificada como la zona con el nivel de desarrollo humano más bajo de las tres. Esta está comprendida por el distrito de Huepetuhe, al lado de la carretera Interoceánica. En cuanto a la salud, la esperanza de vida al nacer es la más

6 Las Piedras y Laberinto, de la provincia de Tambopata; Manu, Madre de Dios y Fitzcarrald, de la provincia del Manu; y Tahuamanu e Ińapari, de la provincia de Tahuamanu.

7 Entrevista a Celso Mendoza, director de Gestión Pedagógica de la Dirección Regional de Educación (DRE) de Madre de Dios. Puerto Maldonado, 1 de marzo de 2017. 
baja en contraste con las otras zonas ( 72,6 años), lo que podría explicarse por la contaminación por mercurio también la afecta, producto de la actividad aurífera ilegal. Si bien no se cuenta con cifras sobre el acceso a fuentes mejoradas de agua, se conoce que las viviendas de los campamentos son muy precarias, dado que estos están en constante movilización en función a la extracción del mineral ${ }^{8}$.

La dimensión educativa, por el contrario, los indicadores PNUD son positivos. Para el año 2012, el distrito de Huepetuhe reveló que el 54,22\% de la población tiene la secundaria completa y los años de educación son 8,73 , cifra que supera a la zona rural. Asimismo, la población de 14 años a más sin nivel educativo es de $19,70 \%$, cifra que también supera a la zona rural. A pesar de que no hay otros indicadores cuantitativos específicos para esta zona, los/as informantes contradicen previamente expuesto: aseguran que «[...] en esas zonas, la capacidad cognitiva de los nińos es limitada»'. En general, es difícil asegurar las cifras en esta zona dada la inaccesibilidad del Estado, que se evidencia en la falta de recolección de datos.

En cuanto a la dimensión económica, el ingreso per cápita es más alto que la zona urbana y el promedio nacional, siendo incluso de los más alto a nivel de todos los distritos del país (S/ 1128,37). Ahora bien, esta cifra proviene de la minería informal/ilegal (60,1 miles de personas que están ocupadas de manera informal) lo que implica que los estándares de trabajo son inexistentes y dicha actividad resulta bastante riesgosa sin las medidas de seguridad mínimas.

Según los indicadores presentados sobre la zona de La Pampa, se podría sugerir un nivel similar al rural, no obstante, estudios empíricos realizados en la zona contradicen estos hallazgos (Mujica, 2014; Vallejo, 2014; Arriarán y Chávez, 2017). Retomando el planteamiento inicial del desarrollo humano, ¿podemos hablar de un entorno en donde las personas son libres, donde tiene posibilidades medias de vivir la vida que desean vivir en La Pampa? Debe resaltarse que una las características más resaltantes de la zona es la trata de personas, que afecta en su mayoría a jóvenes, tanto mujeres como varones: explotación sexual y laboral, respectivamente (Mujica, 2014). Esta actividad se gesta alrededor de la minería ilegal y mientras esta no se erradique, resulta difícil que la trata cese. En cuanto a esta actividad, las condiciones de salud de las víctimas son desconocidas, a excepción de algunos casos registrados y se sabe que la tasa de reinserción escolar de víctimas es mínima, siendo las mujeres las más afectadas en la zona (Vallejo, 2014).

En suma, si bien los indicadores señalan un nivel medio de desarrollo humano, distintos estudios, testimonios y reportajes periodísticos contradicen esto,

8 Entrevista a Yula Rojas, directora de Salud de las Personas de la Diresa. Puerto Maldonado, 28 de febrero de 2017.

9 Entrevista a Celso Mendoza, director de Gestión Pedagógica de la DRE. Puerto Maldonado, 28 de febrero de 2017. 
introduciendo problemáticas que contradicen la definición de desarrollo humano. De esta manera, se observa un nivel de desarrollo humano bajo y además que afecta de manera desigual a los pobladores de esta zona.

Resumiendo, en el caso de la zona urbana, ampliar las libertades resulta una posibilidad, al poseer un nivel desarrollo humano alto, aunque divergente entres sus habitantes. La zona rural, por su parte, ampliar las libertades puede significar mucho más esfuerzo por parte de las personas, dado el nivel inferior, aunque uniforme y parejo. En el caso de La Pampa, para muchas personas ampliar las libertades es imposible, porque no existe libertad y para el resto de personas, el entorno no es favorecedor para promover el desarrollo humano. Resulta claro el contraste en el desarrollo humano de estas tres zonas y la importancia de explicar a estas diferencias.

\section{EL COSTO DEL BIENESTAR: EXPLICANDO DIFERENCIAS TERRITORIALES}

La presente sección busca evidenciar las relaciones entre Estado y sociedad civil que tienen como producto los distintos niveles de desarrollo humano propuestos previamente. Dicho análisis será llevado a cabo a partir de la literatura existente, la prensa escrita y el trabajo de campo realizado.

En pocas palabras, las zonas con distinto nivel de desarrollo humano son explicadas por distintas relaciones Estado-sociedad, lo que contribuye a una adecuada distribución de los servicios de salud y educación, lo que contribuye directamente al desarrollo humano de las personas y permite la expansión de sus posibilidades y libertad.

En el caso de la zona urbana, la relación Estado-sociedad tiene la forma de una coalición, en donde actores provenientes de la sociedad civil organizada y funcionarios del Estado trabajan en conjunto en favor de la provisión de servicios sociales a la población. Asimismo, esta coalición tiene instalada más de treinta años de trabajo en la región, por lo que tiene estrategias previamente practicadas y conocidas, así como aliadas/os dentro de las instituciones relacionadas a los rubros. En el caso de la zona rural, a pesar de que la coalición proveniente de la zona urbana tiene una intermitente injerencia en la zona, el esfuerzo no es suficiente para que el nivel se iguale. Las acciones concretas son de fiscalización y protesta por parte de la sociedad civil organizada, pero aún así, la intervención del Estado es mínima, principalmente debido a la falta de presupuesto. En el caso de la zona de La Pampa, el nivel de desarrollo humano es bajo, pues la relación Estado-sociedad en esta zona tiene la forma de desafiantes territoriales, lo que desincentiva al Estado de intervenir y bloquea el desarrollo humano. 
Tabla 1. Metodología del desarrollo humano en Madre de Dios

\begin{tabular}{|c|c|c|c|c|c|c|c|c|}
\hline $\begin{array}{l}\text { Dimen- } \\
\text { sión }\end{array}$ & Indicador & Año & Unidad & Nacional & Regional & Urbana & Rural & Ilegal \\
\hline \multirow{4}{*}{ 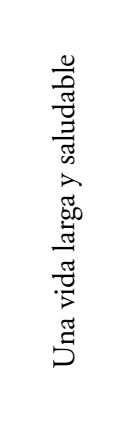 } & $\begin{array}{l}\text { Esperanza de vida } \\
\text { al nacer }\end{array}$ & 2012 & Años & 74,31 & 72,31 & 80,23 & 70,06 & 72,60 \\
\hline & $\begin{array}{l}\text { Anemia (niñas y } \\
\text { niños entre } 6 \text { y } 36 \\
\text { meses) }\end{array}$ & 2015 & $\%$ & 35,6 & 58,20 & 58,20 & 58,20 & 58,20 \\
\hline & $\begin{array}{l}\text { Acceso a fuentes } \\
\text { mejoradas de agua }\end{array}$ & 2011 & $\%$ & - & - & 80,00 & 32,00 & - \\
\hline & $\begin{array}{l}\text { Contaminación por } \\
\text { mercurio }\end{array}$ & 2016 & & $\mathrm{NO}$ & - & $\mathrm{NO}$ & SI & SI \\
\hline \multirow{4}{*}{ 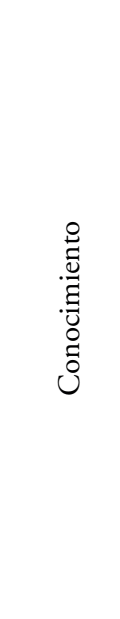 } & $\begin{array}{l}\text { Población con edu- } \\
\text { cación secundaria } \\
\text { completa }\end{array}$ & 2012 & $\%$ & 67,87 & 61,66 & 72,21 & 35,03 & 54,22 \\
\hline & $\begin{array}{l}\text { Años de educación } \\
\text { (Poblac. } 25 \text { y más) }\end{array}$ & 2012 & Años & 9,00 & 8,94 & 10,71 & 8,17 & 8,73 \\
\hline & $\begin{array}{l}\text { Pruebas ECE } \\
\text { (alumnos de } 2^{\circ} \\
\text { de secundaria que } \\
\text { alcanzaron el nivel } \\
\text { suficiente en la } \\
\text { prueba matemática) }\end{array}$ & 2016 & $\%$ & 11,5 & 7,4 & 7,90 & 2,90 & - \\
\hline & $\begin{array}{l}\text { Población de } 14 \\
\text { años a más sin nivel } \\
\text { educativo }\end{array}$ & 2013 & $\%$ & - & - & 10,00 & 21,30 & 19,70 \\
\hline \multirow{2}{*}{ 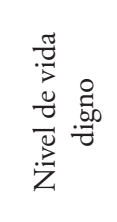 } & $\begin{array}{l}\text { Ingreso familiar per } \\
\text { cápita }\end{array}$ & 2012 & $S /$. & 696,9 & 1011,3 & 1053,03 & 624,80 & 1128,37 \\
\hline & $\begin{array}{l}\text { Población ocupada } \\
\text { por empleo formal } \\
\text { y empleo informal }\end{array}$ & 2015 & $\begin{array}{l}\text { Miles de } \\
\text { personas }\end{array}$ & 15796,9 & 79,0 & 18,9 & \multicolumn{2}{|c|}{60,1} \\
\hline
\end{tabular}

\subsection{Puerto Maldonado, la ciudad de las oportunidades}

Puerto Maldonado es la zona con el nivel de desarrollo humano más alto en Madre de Dios, en donde el Estado prevé de servicios sociales básicos a toda la población, tales como de salud y educación, aunque de manera heterogénea o desigual, como se explicó en la sección anterior. Este nivel se sustenta por la relación Estado-sociedad que acontece en la esta zona: una sociedad civil organizada que, en conjunto con el Estado, forman una coalición que busca promover el desarrollo humano, lo que 
facilita la intervención del Estado. Adicionalmente, la existencia de gran cantidad de servicios sociales privados también interviene para mejorar el nivel de desarrollo humano de esta zona. Como resultado, el nivel de desarrollo humano es alto.

Como se mencionó previamente, la figura de la coalición será clave en el caso de la zona urbana: la Mesa de Concertación contra la Pobreza (MCLCP) representa la organización de la sociedad civil, en conjunto con instituciones del Estado para promover el desarrollo humano de distintas maneras. Así, representa una coalición en la zona urbana, según las características de Sabatier y Jenkins-Smith (1993).

La MCLCP está ubicada en el centro de la ciudad de Puerto Maldonado y consta de dos oficinas a donde asisten a diario las tres coordinadoras voluntarias. Una misionera dominica (como la principal coordinadora), un abogado voluntario y una secretaria. Ellas organizan las sesiones periódicas a través de convocatorias dirigidas a los miembros que conforman la Mesa, para así tratar los temas referentes a distintos temas que afectan a la población y formular soluciones ${ }^{10}$. El éxito de esta mesa sustenta en las siguientes características:

Primero, como coalición, está conformada por miembros diversos que logran converger en intereses, pertenecientes a la sociedad civil organizada y de las instituciones del Estado. Estos representantes son designados por sus propias instituciones y solo ellos tienen derecho a un voto durante las sesiones. De las instituciones del Estado, están presentes el gobierno regional (Direcciones Regionales de Educación, Salud, Trabajo, Agricultura, etc.), del gobierno central (Dirección Desconcentrada de Cultura, Policía Nacional del Perú, Centro Emergencia Mujer [CEM], etc.) e instituciones autónomas (Defensoría del Pueblo, Universidad Nacional), donde las instituciones con mayor participación provienen del sector educación, salud y la Defensoría del Pueblo. De las instituciones provenientes de la sociedad civil, están ONG (medioambientales, sociales, etc), Colegios de Abogados, Ingenieros, etc., la Fenamad e instituciones religiosas. Su participación activa varía según el tema que se trate durante las sesiones. La MCLCP se organiza a partir de mesas de trabajo temáticas, en las cuales se agrupan las instituciones concernientes al tema y cualquier otra institución interesada. A partir de la discusión y toma de decisiones sobre los temas, la Mesa se organiza para realizar desde campañas de concientización hasta demandas y reclamos al gobierno regional o el gobierno central y sus instituciones residentes en Lima. Así, esta coalición conserva sus objetivos y a partir de las sinergias entre la sociedad civil y el Estado, logran hacer efectiva la intervención de este último.

Segundo, la dirección de la Mesa es clave para su funcionamiento: la hermana Lourdes (misionera dominica) es miembro del Vicariato, lo que la vuelve una persona

10 Entrevista a la hermana Lourdes, coordinadora de la MCLCP. Puerto Maldonado, 28 de octubre de 2017. 
respetable por el resto de actores y ciudadanos/as, lo que tiene explicación en la historia de la región, en donde el Estado estuvo ausente y la Iglesia católica, a través de sus misioneros y misioneras dominicos/as, se encargaron del bienestar (gestión de colegios, campañas de salud) en la región. Tercero, los políticos no participan en la Mesa. Como se mencionó previamente, Madre de Dios es una región en la que los líderes políticos están vinculados directamente en su gran mayoría con la minería, por lo que le dan interés a temas relacionados con este sector. Por lo tanto, son los y las funcionarias quienes trabajan en la MCLCP en vez de autoridades de elección popular, lo que asegura la continuidad de la coalición. En suma, la composición, la dirección y la falta de politización de la coalición resultan elementos que contribuyen al funcionamiento óptimo de esta coalición, que tiene como objetivo la promoción del desarrollo humano, a través de la fiscalización y proposición para la provisión de servicios de bienestar, para que así, estos sean óptimos.

Ahora bien, ¿`cómo se logra un nivel alto de desarrollo humano? En cuanto a la fiscalización, se buscan la sinergia con el Estado para asegurar la adecuada provisión de servicios sociales básicos, a través de la mesa de trabajo de su competencia:

[...] existen carencias en el Gobierno Regional... La infraestructura educativa, hay colegios acá en la ciudad, aulas que justamente mañana vamos a ir a inspeccionar en una reunión con el Gerente General, aulas que no tienen pared, que no tienen ventana, solo tienen techo, parte de la pared, sin sillas, sin mesas y estamos a dos semanas del inicio de las jornadas escolares. Nosotros mańana vamos a hacer una inspección y si es que no se ha hecho nada, porque la semana pasada hicimos inspección y les hicimos conocer esta situación al director de la Dirección de Educación para que ellos adopten opciones para que esto mejore, sino vamos a mandar al Ministerio Público por omisión de funciones, porque es responsabilidad del Estado, a través del gobierno regional y a través de la Dirección Regional de Educación garantizar que esas aulas estén operativas ${ }^{11}$.

Ahora bien, este tipo de acciones no son únicas de la zona urbana. Como se dijo previamente, la zona urbana, al ser capital, es el centro de distintos tipos de negociaciones, por lo que, las negociaciones de la MCLCP tienen impacto, aunque con menor magnitud en otras zonas, aspecto que se profundizará más adelante.

Por último, como se señaló con anterioridad, los servicios sociales privados, tales como las clínicas, farmacias y colegios privados juegan un rol importante en el bienestar, relacionado, además, con la concentración del capital económico en Puerto Maldonado. Básicamente, las personas con más ingresos tienen más posibilidades de acceder a servicios sociales privados, un lujo que no existe en la zona rural.

11 Entrevista a Guimo Loayza, Defensor del Pueblo. Puerto Maldonado, 1 de marzo de 2017. 
En conclusión, la zona urbana goza de un nivel alto de desarrollo humano gracias a la figura de la coalición y los servicios sociales privados. La coalición, como la MCLCP, funciona como un espacio que se mantiene en el tiempo de diálogo entre actores pertenecientes al Estado y la sociedad civil, con el fin de promover el desarrollo humano y acabar con la pobreza, a través de la fiscalización constante y la formulación de propuestas. Asimismo, es importante no olvidar el rol que tienen los servicios sociales privados para lograr un alto desarrollo humano en la zona.

\subsection{Ruralidad sin importancia}

La zona rural, la cual se reparte alrededor de siete u ocho distritos a lo largo de la región, es la zona con el nivel mediano de desarrollo humano. La configuración de Estado-sociedad en esta zona tiene muchas formas. Por un lado, la coalición, la cual tiene lugar en la zona urbana, tiene injerencia, de manera intermitente en varios distritos de la zona rural. Por su parte, otras organizaciones, no necesariamente vinculadas a la MCLCP, funcionan como sustitutos del Estado, para proveer de servicios de salud y educación a la zona.

Existen dos estrategias clave de las relaciones Estado-sociedad, la cual tiene la forma de coalición, pero mucho más débil. En primer lugar, la MCLCP tiene una mesa que busca promover la identificación de las personas en Madre de Dios, frente al gran problema de la falta de identificación de un gran porcentaje de la población: «[...] en el 2015 se constituyó una mesa de trabajo por la identificación de los ciudadanos [...] sin DNI no hay derechos. Gente sin DNI que quiere atenderse por salud, lo primerito que le piden es su DNI. El SIS sabemos que les da dos meses de una inscripción temporal, pero eso no quiere decir que te vas a sanar en dos meses. ¿Y después?». La mesa de trabajo busca erradicar esta situación a través de la realización de campañas de sensibilización dirigidas al Estado, demandando la instalación de una sede regional del Registro Nacional de Identificación y Estado Civil (Reniec) en la región. Estas están dirigidas a las personas para informarles de los beneficios del DNI, imprescindible para acceder a servicios de salud (los establecimientos de salud solicitan el documento de identificación para la atención) y educación (los niños y niñas sin DNI no puede ser registrados en el servicio del Ministerio de Educación para las notas).

En segundo lugar, frente al problema de la falta de capacidad del Estado para proveer el servicio educativo, se realiza la "gestión compartida", un convenio que realiza el Estado con la Fenamad y el Vicariato, por separado. Este consiste en un intercambio: estas organizaciones ofrecen apoyo en la gestión de colegios en la zona rural (brindar plataformas de capacitación a los docentes de Educación Intercultural Bilingüe [EIB], así como espacios para que estos realicen el registro de notas, etc.), pues el Estado no puede brindar este servicio mientras que las 
instituciones demandan poder en estas instituciones (elegir a los docentes y directores de las instituciones educativas [IIEE] que pertenecen al convenio) ${ }^{12}$.

La Fenamad, por su parte, tiene una larga tradición en Madre de Dios, al reivindicar los derechos de los ciudadanos originarios (Paredes, 2015). Al igual que el caso del Vicariato, la Fenamad se impone frente a una problemática de dotación del servicio, pues estas no pueden otorgar los servicios de manera óptima ${ }^{13}$. Entonces, el intercambio de la gestión compartida con la Fenamad se da de la siguiente manera: la Federación tiene la facultad de participar en la contratación de docentes y directores de los colegios que gestiona, que son alrededor de veinte colegios a nivel rural. A cambio de este poder, capacita a los docentes en la modalidad de EIB, aspecto en el que la DRE encuentra dificultades. De esta manera, frente a la falta de interés y de capacidad que demuestra el Estado, distintas organizaciones logran coordinar y presionar a este para mejorar la dotación de servicios de educación y salud. Esto explica el nivel de desarrollo humano de la zona rural que, por lo revisado, logra superar las adversidades en cuanto a la dimensión educativa, mas no hace frente a la falta de atención del sector salud. Por ello, el nivel de esta dimensión es menor, calificado como medio.

\section{3. «Tierra de nadies»}

La Pampa es la zona de Madre de Dios en la que se concentra actividad minera informal e ilegal. Previo al análisis respectivo, vale destacar dos características que condicionan el desarrollo humano en cada nivel de análisis.

Primero, si bien la migración es un fenómeno que afecta en gran medida a toda la región, en La Pampa la migración es masiva, al punto que hay un consenso entre todos los entrevistados de una asociación inmediata entre La Pampa y una «población flotante», a modo de una convención social dentro de la región para explicar que la población es mucha pero el Estado no la contabiliza debido a su constante movilización ${ }^{14}$. Este fenómeno se debe a la actividad minera, explicada extensivamente en el capítulo I. Esta característica condiciona el desarrollo humano en el sentido de la dotación de los servicios.

Segundo, La Pampa se diferencia del resto de zonas por una característica en particular: no existe un Estado de derecho, ni proveniente del nivel nacional o subnacional, lo que se puede observar claramente en el Mapa 2, en donde se observa

12 Entrevista a Mariela Reyna, coordinadora de la Red Escolar de la Selva del Sur Oriente Peruano (Resop). Puerto Maldonado, 27 de octubre de 2017 y entrevista a la Hermana Lourdes, coordinadora de la MCLCP. Puerto Maldonado, 28 de octubre de 2017.

13 Entrevista a Julio Cusurichi, presidente de la Fenamad. Puerto Maldonado, 2 de marzo de 2017.

14 Entrevista a Consuelo Cano, coordinadora de la Encuesta Nacional de Hogares Madre de Dios. Puerto Maldonado, 28 de febrero de 2017. 
cómo se distribuye la provisión de servicios (establecimientos de salud e IIEE) alrededor de la zona y solo unos pocos establecimientos dentro de ella. Esta particularidad precondiciona la presencia del Estado y su posibilidad de proveer servicios sociales básicos a la población que habita allí, puesto que es incapaz de ingresar a esta zona. De hecho, este mapa logra ilustrar las tres zonas de desarrollo humano, a partir de la distribución de servicios sociales.

Mapa 2. Distribución de servicios (salud y educación) en Madre de Dios y ubicación física de las zonas planteadas

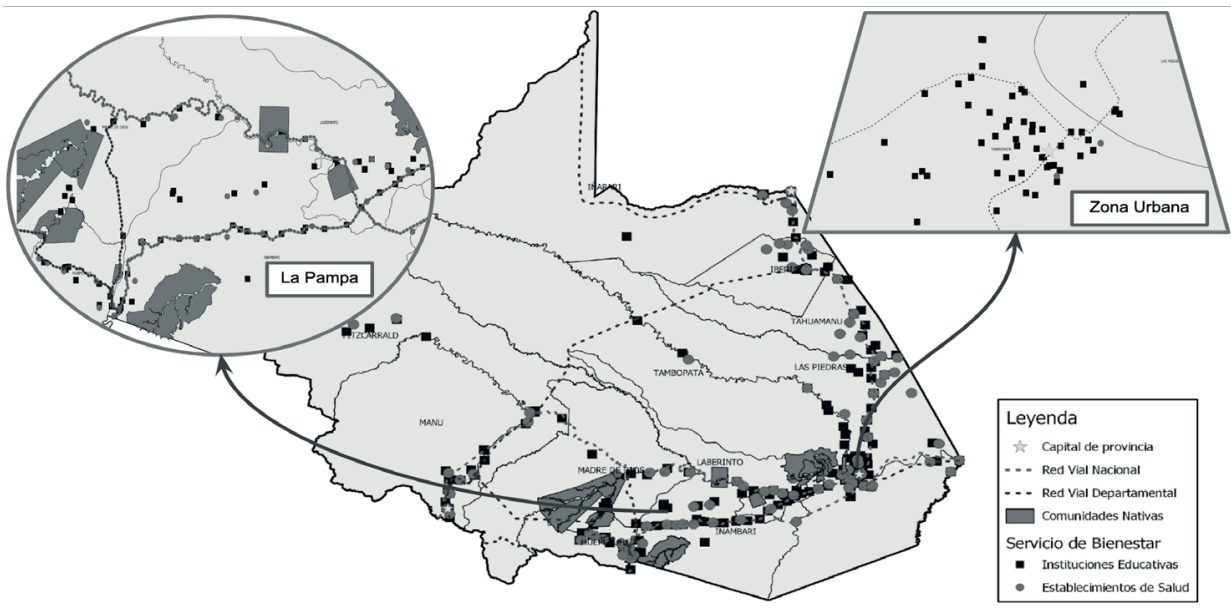

Fuente: Fuentes, 2018. Elaboración propia.

Como se dijo previamente, la zona marcada por situaciones de ilegalidad posee el nivel de desarrollo humano más bajo de la región de Madre de Dios debido a la configuración de desafiantes territoriales, lo que condiciona la intervención del Estado, pero a la vez tampoco hay capacidad de ingreso a la zona (Eaton, 2012). Sin embargo, también se configuran coaliciones que superan las adversidades y buscan promover el desarrollo humano. La MCLCP y el Centro de Promoción y Defensa de los Derechos Sexuales y Reproductivos (Promsex), como coaliciones trabajan en esta línea, a diferencia del primer grupo, no interesado en el tema del bienestar en lo absoluto.

Sobre los desafiantes, La Pampa posee dos tipos de desafiantes territoriales del Estado: la Fedemin y las bandas criminales de mineros ilegales, no necesariamente excluyentes entre sí, pues defienden la misma actividad. Además, los pobladores de esta zona no necesariamente son parte de estos grupos, pero sí se dedican a la actividad aurífera y se oponen también a su erradicación. El conjunto de estos actores resulta un desincentivo para el Estado. En el decreto legislativo 1100 se plasman los intereses de la Fedemin, el que regula la interdicción de la minería ilegal en la zona de La Pampa. Asimismo, los grupos criminales tienen una lógica que consiste en 
prevalecer el sistema de La Pampa. En ese sentido, cualquier intento de perturbar el orden interno, es sancionado por ellos. Así, se muestran casos de brutales asesinatos a quienes revelan la situación interna de La Pampa (Arriarán y Chávez, 2017).

Ahora, ¿cómo estos actores desincentivan la presencia del Estado? Su férrea oposición y el deseo de evadir conflictos por parte del Estado dialoga con la propuesta de Holland (2016), en donde se condiciona la presencia y capacidad del Estado, frente a un conflicto o sobre esfuerzo que pueda realizar para lograr intervenir (Slater y Kim, 2015). Esto se evidencia en las distintas intervenciones registradas en los últimos años en La Pampa en donde se observa la quema de máquinas para la actividad. No obstante, meses después se recuperan y la intervención adquiere una connotación simbólica. Observamos una zona sin comisarías, sin sedes del gobierno central ni del regional, solo un espacio de producción aurífera, en donde las posibilidades de tener una vida digna, más allá de los ingresos económicos, son nulas o mínimas. Al no haber estos locales, tampoco encontramos funcionarios, personal de las Fuerzas Armadas o la Policía Nacional del Perú.

El Estado, aunque solo desde los sectores salud y educación, la MCLCP y algunas ONG hacen frente a esta situación y procuran brindar bienestar desde sus posibilidades en los ámbitos sociales (educación y salud) dentro de la zona: por un lado, desde el Estado (específicamente, dentro en los establecimientos que están en cerca a la la zona, como se ve en el Mapa 2), se trata de atender a los niños y niñas, buscando integrarlos/as a las instituciones educativas ${ }^{15}$. En segundo lugar, la MCLCP, como se mencionó anteriormente, trabaja a través de una mesa de trabajo contra la trata de personas activamente hace dieciocho ańos. Esta coalición permanece en el tiempo gracias a la coyuntura y a las instituciones que participan activamente (CHS Alternativo y Promsex) y se oponen a esta actividad ilegal y ponen en agenda el tema de la trata de personas: se realizan campañas informativas en las IIEE y en general, con el fin de informar a jóvenes y prevenir esta situación. Promsex también realiza campañas médicas móviles en conjunto con la Dirección Regional de Salud (Diresa), para acceder a esta zona.

Por último, en La Pampa interviene el factor de los servicios sociales privados, en particular en el sector salud. Esto se manifiesta a través de la presencia de farmacias y algunos médicos particulares que atienden a las personas en la zona, lo que explica un ligero aumento en el nivel de desarrollo humano. Sin embargo, esta variable no es suficiente para influir en el nivel de la zona.

En suma, La Pampa se propone como una zona sin presencia del Estado, lo que limita las posibilidades de acceder a una vida digna, así como incrementar el nivel de desarrollo humano en la zona. Esto se debe a la falta de interés del Estado de

15 Entrevista a Luis Alberto Rodrigo, director de la DRE de Madre de Dios. 23 de febrero de 2017. 
intervenir, a excepción de algunas instituciones de carácter social (salud y educación), aunque de manera débil. No obstante, observamos la figura de la coalición en muchas ocasiones, conformada por actores estatales y no estatales, que buscan suplir esta falta de promoción del Estado del desarrollo humano. Si bien sus esfuerzos no son suficientes, debido a la complejidad del territorio, la coalición se mantiene, así como otras instituciones que trabajan de manera particular, pero constante.

\section{CONCLUSIONES}

Esta investigación se planteó como objetivo explorar un patrón regional de desarrollo humano, con el fin de explicar por qué una región, al parecer con un nivel de desarrollo humano alto y homogéneo, mostraba grandes diferencias en el interior. Una vez planteadas tres zonas con distintos niveles de desarrollo humano en el territorio, el análisis consistió en explorar las relaciones entre el Estado y la sociedad que se gestan en cada una de estas. De esta manera, la investigación se construye sobre la base de dos objetivos: primero, caracterizar y evidenciar las diferencias entre las zonas y segundo, estudiar las relaciones Estado-sociedad dentro ellas, las cuales tienen como producto el nivel de desarrollo humano.

Al caracterizar Madre de Dios, se observan dos caras. La primera aparenta ser una región modelo del desarrollo económico. Este modelo se sustenta en sus indicadores "milagrosos», aquellos que la posicionan como una de las regiones en donde sus habitantes tienen un nivel de vida digno, mientras que la segunda se caracteriza por los altos índices de violencia. Frente a esta disyuntiva, una alternativa es abordar el caso como una región que supera las adversidades y promete una vida decente a sus habitantes. Una simple respuesta consiste en que el desarrollo humano o el bienestar se construye a partir de un capital económico: a pesar de la violenta coyuntura, los habitantes, a partir de sus ingresos económicos pueden acceder a un nivel de vida digno.

Más allá de esta lógica, esta investigación apostó por una mirada cualitativa subnacional, en donde se observan distintos patrones de desarrollo al interior de la región. La investigación parte de una definición del desarrollo humano que le da importancia al espacio territorial en donde se desarrollan las personas, el rol del Estado y a los actores involucrados, ideal para el caso de Madre de Dios.

La investigación demostró que existen tres zonas con distintos niveles de desarrollo humano. Primero, la zona urbana y de más alto nivel de desarrollo humano, ubicada en la ciudad de Puerto Maldonado, el centro económico y político de la región. Dentro de esta, se generan brechas en el nivel de vida de los ciudadanos, lo que hace que esta zona sea de nivel desigual/heterogénea. Es decir, si bien muchos gozan de un nivel de vida alto, otros, en particular en los suburbios, se diferencian 
de estos con un nivel de vida inferior. Segundo, se identificó que la zona rural tiene un nivel de desarrollo humano intermedio y uniforme. Tercero, la zona de La Pampa o la zona minera se caracteriza por tener el nivel más bajo de desarrollo humano y además, desigual.

Habiendo caracterizado cada una de las zonas y evidenciado el nivel de desarrollo humano, se estableció un punto de partida: el Estado como encargado de proveer las condiciones necesarias para asegurar un nivel alto de desarrollo humano. Es decir, se habla de la capacidad que tiene este para proveer los servicios sociales básicos (establecimientos de salud y colegios), lo que se ve condicionado por la relación que el Estado pueda entablar con la sociedad. Estos actores, como demuestra la investigación, también tienen un rol fundamental en la provisión de estos servicios y cómo afecta la relación en el nivel del desarrollo humano.

Así, se plantean cuatro puntos que fueron fundamentales para la investigación. Primero, el Estado puede mostrar interés o no en intervenir en el territorio, más allá de su capacidad. Segundo, se descarta al Estado como único actor involucrado con el desarrollo humano de la población. Frente a un Estado incapaz y desinteresado, dependiendo de la zona, surgen actores no estatales como fiscalizadores en el proceso de la promoción del desarrollo humano, a través de la provisión de servicios sociales básicos (salud y educación). Tercero, estos niveles de análisis evidencian la complejidad de relaciones que se establecen entre Estado y sociedad: los actores no estatales con poder en el territorio no necesariamente cooperan con el Estado. Y así como existen los retadores, existe la coalición, capaz de mantenerse en el tiempo, a pesar de los cambios de gobierno y buscar conseguir su objetivo: erradicar con la pobreza.

Por último, la investigación abre paso al tema de la sostenibilidad de los niveles de desarrollo humano en la región. Es necesario reconocer la labor de las organizaciones no Estatales y en particular, la de la Mesa de Concertación de Lucha Contra la Pobreza, la cual se mantiene gracias a la participación de actores del Estado no-políticos (gobernador, regidores, congresista). Esta tiene influencia en las tres zonas, en mayor o menor medida. Segundo, si bien la despolitización de los actores funciona para la MCLCP, es necesario involucrar a las autoridades de turno, pues el efecto podría ser más inmediato. Por ejemplo, una ley que regule la actividad minera en La Pampa o una interdicción efectiva en la misma. Tercero, es necesario conocer las cifras de la región, a través de un Censo efectivo, que logre cuantificar a la población y así poder brindarle a la misma los servicios que les correspondan. De esta manera, se logrará incrementar el nivel de desarrollo humano y sostenerlo en el tiempo. 


\section{REFERENCIAS BIBLIOGRÁFICAS}

Albina, M (2011) Medición del desarrollo humano a escala territorial: metodología y su aplicación a los casos de Argentina y México. Economía, Sociedad y Territorio, XI(36), 273-315.

Altman, D. y Luna, J. (2012). Introducción: el Estado latinoamericano en su laberinto. Revista de Ciencia Política, 32(3), 521-543. https://doi.org/10.4067/ S0718-090X2012000300001

Amengual, M. (2014). Pathways to enforcement: Labor Inspectors Leveraging Linkages with Society in Argentina. ILR Review, 67(1), 3-33. https://doi. org/10.1177/001979391406700101

Arriarán, G. y Chávez. S. (2017). Espacios de frontera y excepción: encuentros entre los servicios y las necesidades de salud de las mujeres en La Pampa. Lima: Centro de Promoción y Defensa de los Derechos Sexuales y Reproductivos.

Augusto, M. (2016). Los origenes sociales de la institucionalidad politica: una mirada a la gestión ambiental en San Martín (1998-2015) (tesis de licenciatura). Pontificia Universidad Católica del Perú.

Barrantes, R. y Glave, M. (2014). Amazonía peruana y desarrollo económico. Lima: IEP y Grade.

Boone, C. (2012). Territorial Politics and the Reach of the State: Unevenness by Design. Revista de Ciencia Politica, 32(3), 623-641. https://doi.org/10.4067/ S0718-090X2012000300007

Chartock, S. (2013). 'Corporatism With Adjectives'? Conceptualizing Civil Society Incorporation and Indigenous Participation in Latin America. Latin American Politics and Society, 2(55), 52-76. https://doi.org/10.1111/j.1548-2456.2013.00193.x

Cotler, J. (2009). Poder y cambio en las regiones. Lima: IEP.

Dargent, E. y Urteaga, M. (2016). Respuesta estatal por presiones externas: Los determinantes del fortalecimiento estatal frente al boom del oro en el Perú (20042015). Revista de Ciencia Política, 36(3), 655-677. https://doi.org/10.4067/ S0718-090X2016000300003

Eaton, K. (2012). The State of the State in Latin America: Challenges, Challengers, Responses and Deficits. Revista de Ciencia Politica, 32(3), 643-657. https://doi. org/10.4067/S0718-090X2012000300008

Fuentes, M. A. (2018). 'Tierra de nadie': Relaciones Estado sociedad y su efecto en el desarrollo humano en la región amazónica de Madre de Dios (tesis de licenciatura en Ciencia Política y Gobierno). Pontificia Universidad Católica del Perú. Facultad de Ciencias Sociales.

Geddes, B. (1994). Politician's dilemma: Building state capacity in Latin America. Berkeley: University of California Press.

Gerring, J. (2008). Case Selection for case-study analysis: qualitative and quantitative techniques. En J. M. Box-Steffensmeier, H. E. Brady y D. Collier (eds.), The Oxford 
Handbook of Political Methodology for Political Science (pp. 645-684). Oxford: Oxford University Press. https://doi.org/10.1093/oxfordhb/9780199286546.003.0028

Gestión (27 de noviembre de 2016). "El mercurio dańa la salud y el comercio de nativos amazónicos en Perú”. Lima, . https://gestion.pe/economia/mercurio-dana-saludcomercio-nativos-amazonicos-peru-121943-noticia/. Consulta: 17 de Diciembre del 2017

Giraudy, A. (2012). Conceptualizing State Strength: Moving beyond Strong and Weak states. Revista de Ciencia Política, 32(3), 599-611. https://doi.org/10.4067/ S0718-090X2012000300005

Giraudy, A., Moncada, E. y Snyder, R. (2019). Inside Countries: Subnational Research in Comparative Politics. Cambridge: Cambridge University Press.

Hale, H. E. (2006). Why Not Parties in Russia? Democracy, Federalism, and the State. Cambridge, UK: Cambridge University Press.

Herbst, J. (2000). States and Power in Africa: Comparative Lessons in Authority and Control (2a ed.). Princeton, NJ: Princeton University Press.

Holland, A. (2016). Forbearance. American Political Science Association, 110(2), 232-246. https://doi.org/10.1017/S0003055416000083

Instituto Nacional de Estadística e Informática (2016). Evolución de la Pobreza Monetaria 2009-2015. Informe Técnico. Lima: INEI.

Lindberg, S. I., Coppedge, M., Gerring, J. y Teorell, J. (2014). V-Dem: A New Way to Measure Democracy. Journal of Democracy, 25(3), 159-169. https://doi.org/10.1353/ jod.2014.0040

Mann, M. (2008). The Autonomous Power of the State: Its Origins, Mechanisms and Results. European Journal of Sociology, 25(2), 53-64. https://doi. org/10.1002/9780470755686.ch3

Migdal, J. (1997). Studying the State. En M. I. Lichbach y A. S. Zuckerman (eds.), Comparative Politics: Rationality, Culture and Structure (pp. 208-237). Cambridge: Cambridge University Press.

Migdal, J., Kohli, A. y Shue, V. (1994). State Power and Social Forces. Domination and Transformation in the Third World. Cambridge: Cambridge University Press.

Morel, J. (2014). De una a muchas Amazonías: los discursos sobre «la selva». En R. Barrantes y M. Glave (eds.), Amazonía peruana y desarrollo económico (pp. 21-44). Lima: IEP y Grade.

Mujica, J. (2014). Elementos comparados del impacto de la trata de personas en la salud de víctimas adolescentes en el contexto de la minería ilegal de oro en Madre de Dios. Lima: Centro de Promoción y Defensa de los Derechos Sexuales y Reproductivos Promsex.

O’Donnell, G. (1993). Acerca del Estado, la democratización y algunos problemas conceptuales. Una perspectiva latinoamericana con referencias a países poscomunistas. Desarrollo Económico, 33(130), 1-32. https://doi.org/10.2307/3467251

Orihuela, J. (2014). Las barreras al crecimiento económico en Madre de Dios. Lima. 
Pribble, J. (2011). Worlds Apart: Social Policy Regimes in Latin America. Studies in Comparative International Development, 46, 191-216. https://doi.org/10.1007/ s12116-011-9085-0

Programa de las Naciones Unidas para el Desarrollo (2006). Informe sobre desarrollo humano 2005. Washington DC: PNUD.

Programa de las Naciones Unidas para el Desarrollo (2010). Informe sobre desarrollo humano. Perú 2009. Lima: PNUD.

Programa de las Naciones Unidas para el Desarrollo (2012). Informe sobre desarrollo humano 2012. Washington, DC: PNUD.

Programa de las Naciones Unidas para el Desarrollo (2013). Informe sobre desarrollo humano: Perú 2013: Cambio climático y territorio: desafíos y respuestas para un futuro sostenible. Lima: PNUD.

Sabatier, P. y Jenkins-Smith, H. (eds.) (1993). Policy Change and Learning: An Advocacy Coalition Approach. Boulder, CO: Westview Press.

Sartori, G. (1994). Comparative Constitutional Engineering: An Inquiry Into Structures, incentives, and Outcomes. Nueva York: NYU Press.

Sartori, G. (1994). Comparación y método comparativo. En G. Sartori y L. Morlino (coords.), La comparación en las ciencias sociales (pp. 29-50). Madrid: Alianza Editorial.

Scott, J. (1990). Domination and the Arts of Resistance: Hidden Transcripts. Yale University Press. Recuperado de www.jstor.org/stable/j.ctt1np6zz

Selverston, M. (1994). The Politics of Culture: Indigenous Peoples and the State in Ecuador. En D. Van Cott (ed.), Indigenous Peoples and Democracy in Latin America (pp. 131152). Nueva York: St. Martin's Press.

Slater, D. y Kim, D. (2015). Standoffish States: Nonliterate Leviathans in Southeast Asia. Trans Regional and National of Southeast Asia, 3(1), 24-44. https://doi.org/10.1017/ $\operatorname{trn} .2014 .14$

Soifer, H. (2008). State Infrastructural power: Approaches to Conceptualization and Measurement. Studies in Comparative International Development, 3(43), 231-251.

Soifer, H. y vom Hau, M. (2008). Unpacking the Strength of the State: The Utility of State Infrastructural Power. Studies in Comparative International Development, 43(3-4), 219-230. https://doi.org/10.1007/s12116-008-9030-z

United States Agency for International Development (2010). ABC de la descentralización. Lima: Pro Descentralización.

Vallejo, E. (2014). Implicancias de la minería informal sobre la salud de mujeres y niños en Madre de Dios. Cuaderno de investigación SPDA, 13. Lima: Sociedad Peruana de Derecho Ambiental.

Vergara, A. (2015). La danza hostil: poderes subnacionales y Estado central en Bolivia y Perú (1952-2012). Lima: Instituto de Estudios Peruanos.

Ziegler-Otero, L. (2009). Resistance in an Amazonian community: Huaorani organizing against the global economy. Nueva York: Berghahn Books. 